\title{
Téoros
}

Revue de recherche en tourisme

\section{Les Québécois restent de grands voyageurs : une étude de la période 1979-1987}

\section{Jean-Paul Baillargeon}

Volume 8, numéro 3, novembre 1989

URI : https://id.erudit.org/iderudit/1080314ar

DOI : https://doi.org/10.7202/1080314ar

Aller au sommaire du numéro

Éditeur(s)

Université du Québec à Montréal

ISSN

0712-8657 (imprimé)

1923-2705 (numérique)

Découvrir la revue

Citer cet article

Baillargeon, J.-P. (1989). Les Québécois restent de grands voyageurs : une étude de la période 1979-1987. Téoros, 8(3), 36-40. https://doi.org/10.7202/1080314ar d'utilisation que vous pouvez consulter en ligne.

https://apropos.erudit.org/fr/usagers/politique-dutilisation/ 


\section{Introduction}

Le Québec étant une région touristique, les personnes qui la visitent et les equipements pour les accueillir ont fait l'objet de maintes études. Plusieurs de ces analyses ont été axées exclusivement ou en très grande partie sur des préoccupations économiques, cette industrie et sa clientèle comptant désormais pour beaucoup dans la composition du PIB québécois. Assez peu de considérations ont été apportées aux habitudes de voyages des Québécois, à l'exception de ceux qu'ils effectuent au Québec versus hors Québec, manifestement toujours dans une perspective économique, soit la balance touristique, ou encore dans une perspective de marketing, soit ce qui peut les inciter à visiter le Québec'.

Chacun sait que les Québécois voyagent beaucoup?. On a peu analysé jusqu'à présent l'évolution de ces habitudes en tant que telles, surtout lorsqu'il s'agit de voyages internationaux. Quiest allé où et quand? Telle est la question à laquelle cet article veut foumir certains éléments de réponse, à l'aide d'une série de Statistiques Canada produite depuis 1979: Voyages entre le Canada et les autres pays, cat. 66-2013. Nous avons transformé une partie de ces statistiques descriptives en indicateurs de nombres de voyages-personnes par 100000 habitants, de façon à établir le poids relatif de ces voyages selon l'äge et selon les destinations. Pour faire ressortir des tendances, nous avons présenté ces indicateurs sur une base triennale. Une telle approche a permis de neutraliser des effets de conjoncture, comme les variations des taux de change ou des destinations temporairement écartée pour motifs de perturbations politiques ou naturelles. Cette méthode a de plus isolé le phénomène de la récession du début des annés 1980. Nous espérions de la sorte dégager certains aspects sociocul-

"Monsieur Jean-Paul Baillargeonestchercheur al'Institut quebecois de recherche sur la culture. turels reliés aux voyages internationaux des Québécois.

Il en ressort trois traits majeurs quant à l'évolution des habitudes des voyages internationaux des Québécois:

1- les québécois voyagent davantage à l'étranger;

2- cet accroissement tient essentiellement aux personnes les plus âgées;

3- les Québécois ont accru le nombre de leurs visites d'endroits autres que les USA, au détriment de cette dernière destination même si celle-ci demeure toujours préponderante.

\section{Les Québécois les plus âgés sont ceux qui ont accru leurs voyages internationaux}

Globalement, pour 100000 habitants, le nombre de voyages-personnes à l'étranger s'est accru de $1,9 \%$ entre 1979-1981 et 1985-1987. Cet accroissement tient essentiellement aux 45 ans ou plus.

Pour toutes les autres catégories d'âge, le nombre de voyages-personnes par 100000 a diminué. Il y a un rapport certain entre ces évolutions et la façon dont la richesse s'est redistribuée pendant la dernière décennie. En sus de la persistance des inégalités classiques, l'écart entre les âges s'est accentué en faveur des plus âgés, une fraction très importante des jeunes vivant dans des conditions de précarité qui les mettent hors circuit dès qu'il s"agit de consommations autres que les nécessités de la vie".

Ainsi, pour l'ensemble des voyages internationaux, les gens de 45-54 ans ont accru leur taux de départs de 1,7\%, les 55-64 ans de $15,2 \%$ et les 65 ans ou plus de $4 \%$. Il est remarquable de constater que les 55-64 ans sont les seuls à avoir accru leurs taux de voyages à la fois aux USA et dans le reste du monde. Quant aux jeunes, si on fait abstraction des moins de 12 ans qui voyagent en general en compagnie de parents, plus leur insertion dans la vie active est problématique, plus leur taux de voyages a régres- sé. Le niveau moyen de scolarisation étant devenu plus élevé, les jeunes se présentent plus tardivement sur le marché du travail. En conséquence, le groupe qui a perdu davantage de terrain est celui des $20-24$ ans, suivi des $25-34$ ans.

A l'autre extrémité du cycle de vie, il ne faut pas se surprendre de voir les 65 ans ou plus accroître sensiblement leurs habitudes

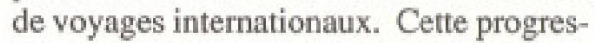
sion vadans la foulée de celle de l'espérance de vie à 65 ans et de l'espérance de vie en santés. En outre, une part croissante de ces personnes atteint l'âge de la retraite dotée d'un fonds de retraite et $\mathrm{d}^{+}$un patrimoine bien garnis s. $^{6}$. Quant à la diminution importante, enfin, du taux des moins de 12 ans, elle peut tenir à deux éléments: les parents $\mathrm{d}^{\dagger}$ enfants de cet âge ont diminué leur fréquence de départs; les parents ayant des enfants de ces åges et qui voyagent le font moins souvent accompagnés de leur progéniture. Les ruptures de mariages et les familles monoparentales sont sans doute pour quelque chose dans ces deux facteurs?

Si on examine maintenant l'évolution de la situation de chaque groupe d'âge par rapport à celle de la moyenne, on constate que seulement deux cohortes ont accru leurs habitudes à un rythme supérieur à celui de la moyenne: les $55-64$ ans $(12,6 \%$ de plus que la moyenne) et les 65 ans ou plus ( 1,3 \% de plus). À l'inverse, la diminution du taux de voyage des jeunes les a éloignés de façon importante de la moyenne, et ce dès les âges les plus bas, la palme allant aux 2024 ans d'abord, les moins de 12 ans les suivant de très près.

Cela vient confirmer le poids considér rable qu'onteu les 55-64 ans dans l'accroissement moyen du taux par 100000 des voyages internationaux des Québécois. Les personnes qui avaient 55-64 ans entre 1985 et 1987 sont nées entre 1921 et 1930 . Cette cohorte, qui a connu une enfance en pleine dépression économique, serait probablement la première génération qui, au terme de sa vie active, a atteint un seuil de revenu suffisant pour se permettre d'accroittre 
TABL RAU

Nombre de voyages-personnes d'une nuit ou plus a 1 exterleur du Canada selon I'Age et la destination, Quebec, 1979-1987

Moyenne triennalo

\begin{tabular}{|c|cccccc}
\hline & $1979-1981$ & & $1982-1984$ & & \\
\hline
\end{tabular}

Sourcet Statistique Cunadi, Voyages entre le Cinadn et les anircs pays. cat. $66-201$, culculs de 1 tateur

considérablement son taux de voyages internationaux, au point d'être pratiquement la seule responsable de la croissance du taux moyen de départs internationaux des Québécois. D'une manière générale, cette population n'a plus de responsabilités financières vis-à-vis ses enfants. Ses dette à long terme sont du passé (hypothèques, etc.). C'est là qu'on trouve les vacances annuelles les plus longues, gräce à l'ancienneté. Ces personnes ont done décidé d'utiliser des surplus de temps et de revenus, parfois très confortables, pour aller voir aussi bien les USA que le reste du monde. Pour plusieurs d'entre elles, c'est souvent leur premier contact concret avec des régions ou des cultures différents, après de multiples années d'images télévisuelles. Cette génération, qui a connu le Québec replié sur lui-même et le monde par la guerre, avait entre 20 et 30 ans lors des premières émissions de télévision. Cette dernière a probablement bouleversé sa vision du monde, sans parler de son rapport avec l'imaginaire. Ayant désomais le temps et les moyens, ces personnes vont voir cet univers qu'on leur a présenté au petit écran, au fil des jours et des années, si différent de celui qu'on leur avait inculqué ou qu'ils avaient pressenti.

\section{Les Québécois ont diversifié leurs voyages internationaux}

L'accroissement du taux général de voyages à l'étranger par 100000 habitants masque uñ phénomène important: la diminution de la part destinée aux USA et l'accroissement de celle vers d'autres ré- gions du monde. En 1979 , près de $87 \%$ des voyages-personnes par 100000 habitants avaient notre voisin du sud comme destination. En 1987, cette part $s^{t}$ approchait de $82 \%$. Malgré cette baisse de popularité, les voyages aux USA demeurent prépondérants. Cela tient à la fois de la proximité de ce pays, de la grande facilité que nous avons de traverser sa frontière et de notre grande familiarité avec cet endroit. Cette familiarité vient de très loin pour plusieurs familles québécoises, l'exode vers la Nouvelle-Angleterre de la fin du XIXe siècle et du début du XXe. Les touristes et les produits américains font partie de notre univers depuis longtemps. Les longs métrages ont êté notre premier contact avec le cinéma ${ }^{\mathrm{g}}$. Notre rapport à cet art reste toujours.très majoritairement tributaire de cette production",

Malgré ces facilités et cette familiarité, il est remarquable de voir que les Québécois ont diversifié leurs destinations internationales quand on sait que chaque voyage ailleursqu'aux États-Unis exige des démarches plus complexes qu'un déplacement vers les USA. Si on peut organiser très facilement un séjour à Old Orchard, une petite visite à Boston ou une fin de semaine à New York, le moindre voyage dans les Antilles ou en Europe prend une autre allure. Malgré ces contraintes, aucun âge n'a échappé au goût pour d'autres paysages, d'autres continents. Ainsi, pour la période observée, les Québécois dans leur ensemble ont accru leur taux de voyages ailleurs qu'aux USA de $46,3 \%$ et ils ont diminué celui à destination de ce pays de $5,3 \%$. En fin de cette période, les 55-64 ans ont voyagé 1,6 fois plus vers ces autres destinations qu'en début, et seulement 1,1 fois plus vers les USA. Les retraités ont accru leur taux de départ pour d'autres continents de 1,5 fois, alor's qu'ils ont diminué quelque peu leur taux de fréquentation des USA. Mais le premier rang revient aux 12-19 ans pour l'accroissement des voyages intercontinentaux: par 100000 , leur taux pour ces destinations s'est accru de près de $58 \%$ entre 1979-1981 et 1985-1987.

Plusieurs ont dô accompagner leurs parents, la plupart de ces derniers se situants vraisemblablement entre 40 et 45 ans. Une partie de ces woyages familiaux doit se composer de Québécois de souche récente, qui sont allés visiter les familles du pays d'origine. On sait par ailleurs que les jeunes de cette cohorte, surtout aux âges les plus avancés, voyagent de plus en plus à l'étranger dans lecadre d'activités parascolaires ou d'échanges (vg. Office francoquébécois pour la jeunesse).

Même si tous les groupes d'âge ont accru leurs taux de voyages ailleurs qu'aux USA, ils ne l'ont pas tous fait dans la meme proportion en regard de la moyenne. Certains ont été davantage favorisés, notamment les 12-19 ans, les 55-64 ans et les 65 ans ou plus. Les activités destinées aux plus jeunes ont certainement contribué au fort écart constaté. Quant aux plus âgés, les facteurs invoqués plus haut à leur endroit se manifestent de façon très marquée par leur situation en regard de la moyenne. Malgré les accroissements de taux dans les autres 
TABLEAU 2

Pourcentage de variation des habitudes de voyage A l'extérieur du Canada selon 1'age et la destination

Quebec, $1985-19871979-1981$

\begin{tabular}{|c|c|c|c|}
\hline Age & Ensemble & aux USA & Alleurs qu'au USA \\
\hline 0.12 & $-7,7$ & -17.5 & +18.7 \\
\hline $12 \cdot 19$ & $-4,2$ & $-9,1$ & +67.6 \\
\hline $20-24$ & $-9,5$ & $-17,5$ & +347 \\
\hline $25-34$ & $-6,2$ & $-12,7$ & +28.7 \\
\hline $35-44$ & $-3,6$ & $-9,8$ & +30.8 \\
\hline 45.54 & $+1,7$ & -58 & +46.3 \\
\hline $55-64$ & $+15,2$ & +84 & +611 \\
\hline $65+$ & $+4,0$ & $+2,5$ & +64.8 \\
\hline moyenne & $+1,9$ & $-5,3$ & +46.3 \\
\hline
\end{tabular}

Source: Calculs a partir du tableau 1

TABLEAU 3

Pourcentage de variation de la situation

par rapport a la moyenne, 1979-1987

$1985-1987 / 1979-1981$

\begin{tabular}{cccc}
\hline Age & Ensemble & aux USA & Allleurs qu'au USA \\
\hline $0-12$ & 10,5 & $-4,9$ & $-20,0$ \\
$12-19$ & $-5,2$ & $-4,8$ & $+10,0$ \\
$20-24$ & $-10,7$ & $-12,7$ & $-7,7$ \\
$25-34$ & $-7,4$ & $-7,7$ & $-11,8$ \\
$35-44$ & 4,9 & $-4,4$ & $-10,1$ \\
$45-54$ & 0,0 & $-0,7$ & 0,0 \\
$55-64$ & $+12,6$ & $+14,3$ & $+10,2$ \\
$65+$ & $+1,3$ & $+2,6$ & $+14,0$ \\
\hline
\end{tabular}

Source: Calculs a partir du tableau 1

groupes d'âge, ces augmentations se situent autour de la moyenne (45-54), ou nettement en-dessous, les 25-34 ans s'en éloignant le plus $(-11,8 \%)$. Là aussi l'effet des plus grandes disparités de revenus a certainement joué, surtout aux âges inférieurs de cett cohorte. Pour ce qui est des $35-44$ ans $(-10,1 \%)$, leur écart par rapport à la moyenne vient aussi, mais à un niveau moindre, des disparités de revenus qui se sont amplifiées entre les plus âgés et les plus jeunes. Quant aux jeunes enfants enfin, encore plus forcément accompagnés de parents lors de voyages intercontinentaux qu'aux USA, leur éloignement de $20 \%$ de la moyenne tiendrait aux raisons deja mentionnées à leur sujet.

Vers où se sont dirigés ces voyageurs lors de leurs visites de pays autres que les États-
Unis? Que ce soit en début ou en fin de période, quatre destinations ont maintenu leur rang. L'Europe vient en premier lieu, suivie des Antilles-Bermudes et des destinations autres (comprenant Saint-Pierre et Miquelon, Océanie, etc.). Plus loin, les croisières et les voyages multi-continentaux conservent le sixième rang et l'Amérique centrale demeure en dernière place. L'Amérique du Sud est devenue un continent plus fréquenté, passant du septième au quatrième rang. $\mathrm{L}^{+}$Afrique et l'Asie, malgré qu'elles aient accru leur popularité, ont perdu de leur importance relative, la première étant passée du cinquième au septième rang, $1^{\top}$ Asie ayant glissée de laquatrième à la cinquiême place.

Toutes ces destinations, sans exception, ont vu croitre leur nombre de voyages-person- nes par 100000 habitants, mais à des rythmes différents. La hausse la plus spectaculaire a été vers l'Amérique du Sud $(+472,7 \%)$. Mais sa part de voyages intercontinentaux en 1985-1987 n'était encore que de $6 \%$, comparativement à $1,5 \%$ en 1979-1981. La seconde en importance a été l'Asie $(+70,7 \%)$. Ce continent ne comptait cependant que pour $3,4 \%$ des voyages internationaux ailleurs qu'aux USA en 19851987 , contre $2,9 \%$ en $1979-1981$. Le troisième accroissement a été celui des croisières et des destinations multiples $(+58,7 \%)$, ces genres de voyages $n^{\dagger}$ ayant été que $2,8 \%$ en $1985-1987$ de tous les voyages autres qu'aux USA et $2,6 \%$ en 1979-1981. Ainsi, les trois principaux accroissements se situent dans des endroits ou des types de voyages assez peu fréquentés, vu leurs coûts élevés. A cet égard, il n'est pas surprenant que l'augmentation des voyages en Afrique n'ait été que de 30,9\%: de plus, ce continent a subi plusieurs soubresauts politiques et on lui a fait une mauvaise réputation en matière de santé. L'Amérique centrale, très peu fréquentée, n"a eu qu'une croissance de $21,7 \%$ de son achalandage de touristes québécois. Cet inter-continent a sûrement souffert là aussi de ses troubles politiques endémiques durant la dernière décennie.

Reste les deux destinations les plus visitées, l'Europe et les Antilles-Bermudes. Le Vieux Continent a maintenu sa part du lion de nos voyages intercontinentaux, $47,3 \%$ en 1979 1981 et $49,8 \%$ en $1985-1987$, près d'un voyage sur deux. En termes de taux de croissance, l'Europe vient au quatrième rang, très près des croisières. Quant aux Antilles-Bermudes, les voyages qu'on y a fait représentaient $30,9 \%$ en 1979-1981 et $24,3 \%$ de l'ensemble de ceux effectués ailleurs qu'aux USA; leur taux de croissance n'a été que de $14,8 \%$. C'est la seule destination qui a connu un recul pendant la récession du début des années 1980.

De tous les endroits visités par les touristes québécois, c'est le seul où l'intérêt quasi exclusif soit le soleil, la mer et la plage.

\section{Conclusion}

Les Québécois voyagent encore beaucoup, aussi bien chez eux et dans le reste du Canada qu'aux USA ou ailleurs ${ }^{10}$. Mais ils ont légèrement délaissé, par 100000 personnes, le Québec, le Canada et les USA pour le reste du monde. Tous ces déplacements d'une nuit ou plus ont représenté une moyenne de 300000 voyages-personnes par 100000 habitants au cours de la dernière décennie, soit environ trois voyages 
TABLEAU 4

Nombre de voyages-personnes internationaux d'une nuit ou plus par 100000 habitants,alleurs qu' aux USA selon la destinationQuebec, 1979-1987

Moyenne triennale

\begin{tabular}{|c|c|c|c|}
\hline & $1979-1981$ & $1982-1984$ & 1985.1987 \\
\hline Europe & 2733 & 2932 & 4211 \\
\hline Afrique & 165 & 195 & 216 \\
\hline Asie & 167 & 192 & 285 \\
\hline Amérique centrale & 23 & 23 & 28 \\
\hline Antilles-Bermudes & 1788 & 1503 & 2053 \\
\hline Amerique du Sud & 88 & 112 & 504 \\
\hline Autres & 666 & 761 & 922 \\
\hline Croisieres et multiples & 150 & 202 & 238 \\
\hline Ensemble & 5781 & 5891 & 8.457 \\
\hline
\end{tabular}

Source: Statistique Canada, Voyages entre le Canada et les autres pays. cat. 55-201, calculs par 1 auteur.

Sourcer. Calculs a partir du tableau 4

par personne par année, dont autour de trois quarts à l'intérieur du Québec, et entre $12 \%$ et $15 \%$ à l'extérieur du Canada, principalement aux USA. On peut considérer que la plupart des voyages à l'intérieur du Québec l'ont été pour des raisons d'affaires ou de famille plutôt que de vacances. L'inverse serait le lot de ceux effectués à l'extérieur des frontières canadiennes. L'augmentation du revenu personnel disponible a forcément favorisé cet engouement pour visiter le reste du monde. Ce goût de "voir du pays" n’est pas particulier aux Québécois; toutes les sociétés industrialisées voyagent maintenant beaucoup.

Mais, quand on sait avec quelle facilité on peut traverser la frontière américaine, il est intéressant de constater que cette destination a perdu de l'attrait au profit d'autres continents. On pratiquerait donc de plus en plus un tourisme de dépaysement, de contacts avec d'autres cultures, d'autres civilisations, au point où la destination soleil des Antilles-Bermudes est celle qui a eu le plus faible accroissement. On recherche encore très largement ce genre de voyages - la popularité accrue des croisières, qui allient mer, soleil et dépaysement en témoigne, mais il n'en reste pas moins que les destinations les plus en croissance vont des mondes qui nous sont le moins familiers à celui avec qui nous avons maintenu des rapports plus affectueux et respectueux que fonc- tionnels ou utilitaires. Comme toute autre population, les Québécois ont pu se laisser séduire par l'exotisme. Mais cet ingrédient a certainement moins joué à l'endroit de l'Europe que de 1'Amérique du Sud ou de l'Asie. Si on fait abstraction, pour ces deux derniers continents, du caractère superficiel qu'on attribue habituellement à l'exotisme, et si on les jumèle à l'Europe, on peut avancer que les Québécois cherchent de plus en plus concrètement à se rapprocher du monde autre qu'anglo-saxon, que son intérêt va vers la diversification de ses contacts culturels, vers la prise de conscience de la grande variété des civilisations. Comme pour échapper à cette sorte de monolitisme linguistique qu'est son environnement nord-américain, comme poursurmontercette solitude culturelle qu'il peut ressentir à l'intérieur de ce continent. Ce genre d'échappée n'est encore accessible qu'à assez peu de personnes et restera sans doute tel. Si environ un Québécois sur deux a fait annuellement un voyage aux USA au cours de la dernière décennie, un sur 17 a fait un voyage ailleurs dans le monde en 1979-1981, mais un sur 12 en 1985-1987. Parmi ces destinations, on a recherché davantage l'expérience à prédominance de découvertes culturelles que celle à prépondérance de bien-être physique et de sensations agréables, sans pour autant avoir mis ces plaisirs au rancart. Le poids des 55 ans et plus y est certainement pour quelque chose; on a moins tendance à ces âges à s'exhiber sur des plages ensoleillées, à faire du surfing ou à côtoyer les "gentils animateurs". Mais cela n'explique pas tout. Sinon l'accroissement des taux de voyages des âges où ces éléments ont plus d'attrait aurait dû se rapprocher du taux de croissance de nos voyages vers cette destination soleil. Quel que soit l'âge, les taux de croissance des voyages intercontinentaux ont été supérieurs à celui à destination des Antilles-Bermudes. L'attrait plus prononcé pour la découverte a donc été supérieur à tout âge à celui pour l'Eden à tarif forfaitaire. $f$ 
Notes explicatives

11) Parmi l'abondance de littérature à ce sujet, nous mentionnons du titre indicatif:

ARCHAMBAULT, Michel, dir- Le tourisme au Québec: bilan et perspectives d'action 1985-1988. Québec, ministére du Tourisme, 1984.

BAUMANN, André et associés L'industrie touristique au Québec: impact bconomique 1970-1980, effectifs, besoins quantitatifs et qualitatifs en main-d'oeuvre, Montréal, André Baumann et associes, 1970 .

BELLEROSE, Pierre, collaboration de Jean PELIETIEA Le libre-echange et le champs récréotouristique: un secteur gagnant, Québec, ministere du Loisir, de la Chasse et de la peche. 1988 .

CAMIRAND, Francois et Gur THIFFAULT, Bilan touristique international en 1978 et son évolution depuis 1972, Québec, ministére du Tourisme, de la Chasse et de la Pêche, 1978 .

CLUZEAU, Patrick, GuY GAUTHIER et Gilles LATERRIERE, Stratégie de développement du loisir touristique, Ouébec, ministére du Loisir, de la Chasse et de la Pêche, 1980.

DEMERS, Jacques, Le tourisme en péril: essai sur le developpement touristique au Québec. Montreal, Nouvelle optque, 1983.

DEMERS, Jacques, dir., "Economie du tourisme": Téores, 7, 3, novembre 1988.

EBACHER, Michel, Les grands facteurs d'attraction touristique au Qubbec; essai, Québec, ministère du Loisir, da la Chasse et de la Péche, 1972.

LAMONDE. Pierre et al. Le tourisme: perspectives de relance: document de réflexion, Québec, Secrétariat des Conférences societeonomiques. 1978.

LAPLANTE, Mare, Jacques JUNEAU et Louise TROTTIER, Gens quipartent et gens qui restent.. les grandes vacances au Québec, Montréal, UOAM, 1983.

STAFFORD, Jean et Marcel SAMSON, "L'industrie touristique quebecoise: entre lo passe et f'woni" " Jean-Paul Baillargeon, dir., Les pratiques culturelles des Québécois: une autre image de nous-mèmes, Québec, IQRC, 1986: 290-317.

(2) CLUZEAU, Patrick, Le Quebec touristique, Ouébec, ministére du Tourisme et Les publications du Quebec, 1987 et 1989.

(3) Cettepublication existedepuis 1920./Mais la notion de voyages-personnes a remplacé celle de nombre de woyages en 1979 . Les données proviennent de "L'Enquête sur les voyages des Canadiens." (EVC). a laquelle le ministere du Tourisme du Québec est associé pour en explaiter les donnêes sur le Québec et les Québécois.

(4) GAUTHIER, Madeleine, L'insertion des jeunes en emploi, Québec. IOAC en préparation.

LANGLOIS, Simon, "Les rigidités sociajes et linsertion des jeunes dans la societe ouebecoise". Fernand Dumont, dir., Une société des jeunes? Québec, IORC, 1986: 301-323.
"Strates er classes sociales: nouveaux riches er nouvaux pauvres: la consommation ". La société québécoise aprés 30 ans de changements, colloque de I'IORC, Québec, 11, 12, et 13 octobre 1989.

MYLES, J., G. PICOT et T. WARNEL, Les salaires et les emplois au cours des années 1980 . évolution des salaires des jeunes et declin de la classe moyenne, Ottawa, Statistique Canada, 1988.

(5) DUCHESNE, Lou's, La situation démographique au Québec, Quebec. Bureau de la statistique du Québec et les Publications du Quebec, edition 1988 .

(6) LANGLOIS, Simon, Ibid.

7) DANDURAND, Renée B., dir., Couples et parents des années quatre-vingt. Question de culture 13. Québec, IORC, 1987 .

DANDURANO, Renee B. et Lise SAINT-JEAN, Des mères sans alliance: monoparentalité et désunions conjugales, Québec, IQRC, 1988.

(8) LAMONDE, Yuan et Pieme.François HEEERT, Le cinéma au Québec: essai de statistique historique: 1986 à nos jours, Quebec, IQRC 1981

(9) BUREAU DE LA STATISTIQUE DU QUEBEC. Projections einématographiques au Québec. 1988, Bureau de la statistique du Québec. 1969.

(10) CLUZEAU, Patrick, tbid.
L'UOAM: Centre international de formation et de recherche en tourisme de l'Organisation Mondiale du Tourisme

Le 2 novembre 1989 , le recteur de l'Université du Québec à Montréal, monsieur Claude Corbo et le secrétaire-général de l'Organisation Mondiale du Tourisme (OMT), monsieur Willibald P. Pahr, ont signé une entente faisant de l'UQAM un Centre international de formation et de recherche en tourisme. Cet accord a été signé en présence du ministre du Tourisme du Québec, monsieur André Vallerand.

L'Organisation Mondiale du Tourisme (affiliée aux Nations Unies) coopérera avee I'UQAM dans tous les domaines de la planification et de la promotion des programmes de formation et de recherche en tourisme, et s'assurera que ces programmes acquièrent une reconnaissance générale. Elle appuiera les demandes de subventions de l'UQAM auprès du gouvernement canadien et des organisations du secteur privé pour créer des bourses d'étude ou d'excellence dans les domaines de la formation et de la recherche en tourisme. L'UQAM profitera aussi d'un accès privilégié au centre de documentation de l'O.M.T.

Pour donner suite à cette entente et déterminer le programme d'activités annuel, un Comité consultatif conjoint sera composé de représentants des organismes suivants: l'OMT, I'UQAM, Tourisme Canada, le ministère du Tourisme du Québec. l'Office des congrès et du tourisme de Grand Montréal, I"Université McGill, l'Institut de tourisme et d'hốtellerie du Québec, la Société du Palais des congrès de Montréal, le Collège Lasalle et le Centre d'études du tourisme.

Source: Module de gestion et intervention touristiques, UQAM 